\title{
Publication Bias: Should Scientists Work Harder to Share Negative Results? Ben Mudrak, Ph.D.
}

Education Division, American Journal Experts

Email: ben.mudrak@journalexperts.com

Scholarly publishing is the cornerstone of advancing our understanding of the world around us. A staggering amount of research is conducted each day, and this research would effectively serve no purpose without a system for its dissemination. However, academic publishing is slanted toward positive results, with negative results becoming scarcer in the literature (Fanelli, 2012). Countless experiments without splashy results end up in dusty lab notebooks, forever closed off from the rest of the scientific community.

The consequences of sheltering negative results are many. First, considerable effort is undoubtedly duplicated by separate labs trying the same experiment, only to generate "uninteresting" results. Second, the focus on only publishing statistically significant results may lead to false conclusions (Ionnides, 2005). In some cases, a chance result may make it into the literature (to be taken as fact) while ten or twenty studies negating that result remain unpublished. This process leads to future studies that may be based on flawed information. Last, important medical decisions hinge on the accurate reporting of clinical trials, which is why recent efforts have focused on registering and publishing all trials, not just those that show favorable or novel results.

A recent study by Suñé et al. in PLOS ONE tracked the publication of results from clinical trials registered at a major hospital in Spain (Suñé et al., 2013). The authors followed 785 studies to completion and were able to find results for 541 (68.9\%). Of these, trials classified as having positive results (i.e., statistically significant effects of the drug being tested) were published $84.9 \%$ of the time. Trials with negative or inconclusive outcomes were published only $68.9 \%$ of the time ( $p<0.001$ compared with positive studies). Moreover, the authors found a longer median time to publication for negative trials compared with positive trials (3.21 years vs. 2.09 years; hazard ratio, 1.99), even when adjusting for the type of sponsorship, the phase of the trial at the time of publication, sample size, and medical area. These results corroborate an earlier meta-analysis of smaller studies on clinical trial reports (Hopewell et al., 2009).

Is this bias caused by journal editors searching for eye-catching results, or authors choosing not to submit negative studies? It's likely to be some of both (Senn, 2013). Unfortunately, the options for publishing negative results are few. The chronic underreporting of negative results in psychology led to the creation of $B M C$ Psychology, which explicitly encourages negative results (Laws, 2013). Otherwise, few journals specifically request negative results, and lower citation rates for negative studies (Jannot et al., 2013) likely make them less desirable to many journals.

The internet has opened up a powerful new avenue for sharing results, but the research community has yet to fully embrace the potential of the web to transform scholarly communication. The internet age provides nearly unlimited storage space, a free exchange of 
information, and new algorithms for data mining. Now should be the right time to create a home for negative results, preventing duplicated effort and ensuring that decisions and conclusions are based on all the available evidence.

\section{References}

Fanelli D (2012) Negative results are disappearing from most disciplines and countries. Scientometrics 90:891-904.

Hopewell S, Loudon K, Clarke MJ, Oxman AD, Dickersin K (2009) Publication bias in clinical trials due to statistical significance or direction of trial results. Cochrane Database of Systematic Reviews. 1:MR000006.

Ionnides JPA (2005) Why most published research findings are false. PLOS Med 2:e124.

Jannot AS, Agoritsas T, Gayet-Ageron A, Perneger TV (2013) Citation bias favoring statistically significant studies was present in medical research. J Clin Epidemiol 66:296-301.

Laws KR (2013) Negativland - a home for all findings in psychology. BMC Psychol 1:2.

Senn S (2013) Authors are also reviewers: problems in assigning cause for missing negative studies. F1000Research 2:17.

Suñé $P$, Suñé JM, Montoro JB (2013) Positive outcomes influence the rate and time to publication, but not the impact factor of publications of clinical trial results. PLOS ONE 8:e54583. 